\title{
Interdisciplinary Issues at the Intersection of Assessing and Treating Substance Use Disorders and Post Traumatic Stress Disorder: Clinical Social Work and Clinical Behavioral Analysis with Veterans
}

\author{
Monica M. Matthieu \\ Alyssa Wilson \\ Robert W. Casner
}

\begin{abstract}
Veterans and military personnel may be at higher risk for developing addictions due to increased prevalence rates of co-occurring mental health disorders including posttraumatic stress and substance abuse disorders. However, clinicians may feel unprepared to assess and to treat these co-occurring disorders, especially when it includes a behavioral addiction such as gambling. Clinical social work and clinical behavior analysis are two fields with complementary interdisciplinary approaches that can lead to improved client-centered outcomes. Yet, limited evidence exists to guide interdisciplinary treatment teams in effective treatment of gambling addictions and Post Traumatic Stress Disorder (PTSD). The current article provides an interdisciplinary treatment model to assist clinicians in selecting appropriate evidence-based assessments and treatments. A case example focuses on the use of assessment tools and treatment approaches drawn from recommendations from best practice guidelines for veterans. Finally, resources related trauma and addictions are presented.
\end{abstract}

Keywords: Post traumatic stress disorder; substance-related disorders; gambling; veteran; United States Department of Veterans Affairs; social work; behavioral disciplines and activities

In 1989, the National Associations of Social Work (NASW) Standards for the Practice of Clinical Social Work defined clinical social work by an emphasis on the process of assessment, diagnosis, intervention, treatment, collaboration, case management, and evaluation of the psychosocial functioning of individuals, families, and small groups (NASW, 1989). As this standard is currently undergoing revision, it can be expected that the collaboration aspect will expand to encompass more modern views on interdisciplinary collaboration with other allied health professions, namely those with similar traditions, theoretical perspectives, and methods. Once such discipline is clinical behavioral analysis. Clinical behavioral analysis is defined as a professional practice that utilizes a similar individually-focused process model to examine, assess, measure, and treat a problematic human behavior. While the main tool is a functional analysis of the behavior, clinical behavioral analysts examine the biological and social forces that impinge on an individual and apply behavioral, cognitive behavioral, and other behavioral medicine methods to instigate change (Association for Behavior Analysis International, 2017).

\footnotetext{
Monica M. Matthieu, PhD, LCSW is Assistant Professor, School of Social Work, College for Public Health and Social Justice, Saint Louis University, Saint Louis. MO 63103. Alyssa N. Wilson, Ph.D., BCBA-D, LBA is an Assistant Professor of Applied Behavior Analysis in the School of Social Work, College for Public Health and Social Justice, Saint Louis University, Saint Louis. MO 63103. Robert W. Casner, LMSW is Doctoral Student in the School of Social Work, College for Public Health and Social Justice, Saint Louis University, Saint Louis. MO 63103. This work does not represent the views of the Department of Veterans Affairs or the United States Government. 
With such common ground as the person-in-environment theoretical perspective, clinical social work and behavior analysis have a special interdisciplinary connectedness that can be helpful in addressing and treating complex behavioral issues. Both disciplines subscribe to a process model for clinical practice that includes five steps (Wilson \& Matthieu, 2015). The steps are: 1) identification of clinically relevant target behaviors; 2) use of reliable and valid measurements; 3 ) relationship between target behaviors, measures, and treatment; 4) data-based treatment decisions; and 5) social validity of treatment process and outcomes.

At present, there are a number of clinically relevant areas of overlap between the two disciplines that are not well known. Some however, are gaining more notice and relevance for extremely high-need clients with behavioral issues, such as children with Autism and individuals with complex clinical presentations requiring behavior based interventions, such as veterans with Post Traumatic Stress Disorder (PTSD) and co-occurring substance use or gambling disorders.

As the Department of Veterans Affairs (VA) expands services to the newest generation of veterans and continues to serve all veterans from military discharge to death, there is a growing need for community providers to understand, address, and treat the biopsychosocialspiritual aspects trauma. While the health and psychosocial needs of veterans are paramount, clinicians need to ensure they have the competencies to support shared decision-making, patient-centered care, and collaborative case management of veteran clients who seek services both in the community and in the VA health care system. In this way, interdisciplinary collaboration, such as that between clinical social work and clinical behavior analysis, can lead to significant client-centered outcomes and changes in health behaviors (Wilson \& Matthieu, 2015). While there are also many barriers to treatment in the community and the VA such as client follow-through, mutual agreement on target behaviors (e.g., who decides on the target behavior during treatment?), and clinician 'choice' of intervention selection when co-occurring conditions are present (e.g., treatment of what condition is primary?), the use of evidence based behavioral practices joins these professionals in the pursuit of psychosocial recovery for complex conditions such as PTSD, substance use, and addictions.

\section{Specific Aims}

The aim of this paper is to provide a framework for clinicians to effectively, and collaboratively, assess and treat PTSD and substance use disorders, in particular gambling. This manuscript will outline a model for interdisciplinary practice to assist social work, behavioral analysis, and other allied health clinicians working with addictions and traumarelated symptoms in selecting appropriate evidence based assessments and treatments. Further, a case example focused on the use of these assessment tools and treatment approaches drawn from recommendations from best practice guidelines from the Department of Veterans Affairs/Department of Defense (DVA/DoD) Clinical Practice Guidelines will be presented for veterans with PTSD and addictive behaviors. Finally, resources related to the training of interdisciplinary treatment teams on trauma and addictions, particularly for behavioral additions such gambling, will be presented. 


\section{Overview of Gambling and Substance Use Disorders}

Gambling is one of the most frequent activities that people engage in around the world. Raylu and Oei (2002) defined gambling as placing value upon a game/event or a bet of any type that has an unpredictable outcome, and in which the result to some magnitude is determined by chance. Gambling occurs in many different forms to include cards, sports betting, casinos, etc. and is participated in widely across socioeconomic levels. Research has demonstrated that gambling was a frequent occurrence in the earliest of societies, and modern games of chance have evolved from the games once played by our ancestors (Bolen \& Boyd, 1968). In the United States, as in many countries, individuals gamble on games and events, and most do so without experiencing problems. Numerous people play games of chance for recreation, however, for some individuals, gambling becomes problematic.

Previous editions of the Diagnostic and Statistical Manual (DSM IV) (APA, 1994, p. 615) defined pathological gambling as a progressive and chronic disorder that encompasses an unrelenting failure to resist impulses to gamble and where this "maladaptive behavior disrupts, or damages personal, family, or vocational pursuits." The negative impact that individuals experience is far reaching. Pathological gamblers have high rates of cooccurring disorders, including mood, personality, and substance use disorders (Kessler et al., 2008; Petry, Stinson, \& Grant, 2005).

The most recent update, DSM V, changed the way pathological gambling is classified. Pathological gambling is no longer viewed as an inability to resist impulses, but rather a substance use disorder (SUD), specifically named, gambling disorder. DSM V (APA, 2013, p. 586) states that "although some behavioral conditions that do not involve ingestion of substances have similarities to substance-related disorders, only one disordergambling disorder - has sufficient data to be included in this section." With regard to gambling being classified as a disorder that is included in this section with substance use, the DSM V (APA, 2013) provides criteria and organization for the associated symptoms. Additionally, DSM V (APA, 2013) now provides specific diagnoses associated with this substance class with regard to SUD. Ten substance classes, relevant to SUD, are highlighted in the DSM V chapter. A side by side comparison of a substance related disorder, alcohol use disorder, and a non-substance related disorder, gambling disorder (GD), highlights the parallel in behavioral symptoms and thus, clarifies the rationale for including GD in the SUD discussion. (See Table 1).

When comparing to the criterion, the rational for including GD in the SUD section of the DSM V becomes evident. For example, SUD criterion states that "the individual may express a persistent desire to cut down or regulate substance use and may report multiple unsuccessful efforts to decrease or discontinue use" (APA, 2013, p. 483). Furthermore, "the individual may spend a great deal of time obtaining the substance, using the substance, or recovering from its effects" (APA, 2013, p. 483). Similarly, individuals who experience GD "need(s) to gamble with increasing amounts of money in order to achieve the desired excitement, has made repeated unsuccessful efforts to control, cut back, or stop gambling, is often preoccupied with gambling" (APA, 2013, p. 585). While diagnostically similar, clinicians with familiarity with the diagnostic criteria now have the foundational knowledge to begin to identify GD, however additional training in a clinical process that 
blends identification, assessment, and treatment may be necessary to ensure competency when gambling may co-occur with other conditions.

Table 1. Comparison of Diagnostic Criteria for Gambling and Alcohol Use Disorder.

\begin{tabular}{|c|c|}
\hline Gambling Disorder Criteria & Alcohol Use Disorder \\
\hline $\begin{array}{l}\text { 1. Needs to gamble with increasing amounts of } \\
\text { money in order to achieve the desired } \\
\text { excitement. } \\
\text { 2. Is restless or irritable when attempting to cut } \\
\text { down or stop gambling. } \\
\text { 3. Has made repeated unsuccessful efforts to } \\
\text { control, cut back, or stop gambling. } \\
\text { 4. Is often preoccupied with gambling (e.g., } \\
\text { having persistent thoughts of reliving past } \\
\text { gambling experiences, handicapping or planning } \\
\text { the next venture, thinking of ways to get money } \\
\text { with which to gamble). } \\
\text { 5. Often gambles when feeling distressed (e.g., } \\
\text { helpless, guilty, anxious, depressed). } \\
\text { 6. After losing money gambling, often returns } \\
\text { another day to get even (“chasing" one's losses). } \\
\text { 7. Lies to conceal the extent of involvement with } \\
\text { gambling. } \\
\text { 8. Has jeopardized or lost a significant } \\
\text { relationship, job, or educational or career } \\
\text { opportunity because of gambling. } \\
\text { 9. Relies on others to provide money to relieve } \\
\text { desperate financial situations caused by } \\
\text { gambling. }\end{array}$ & 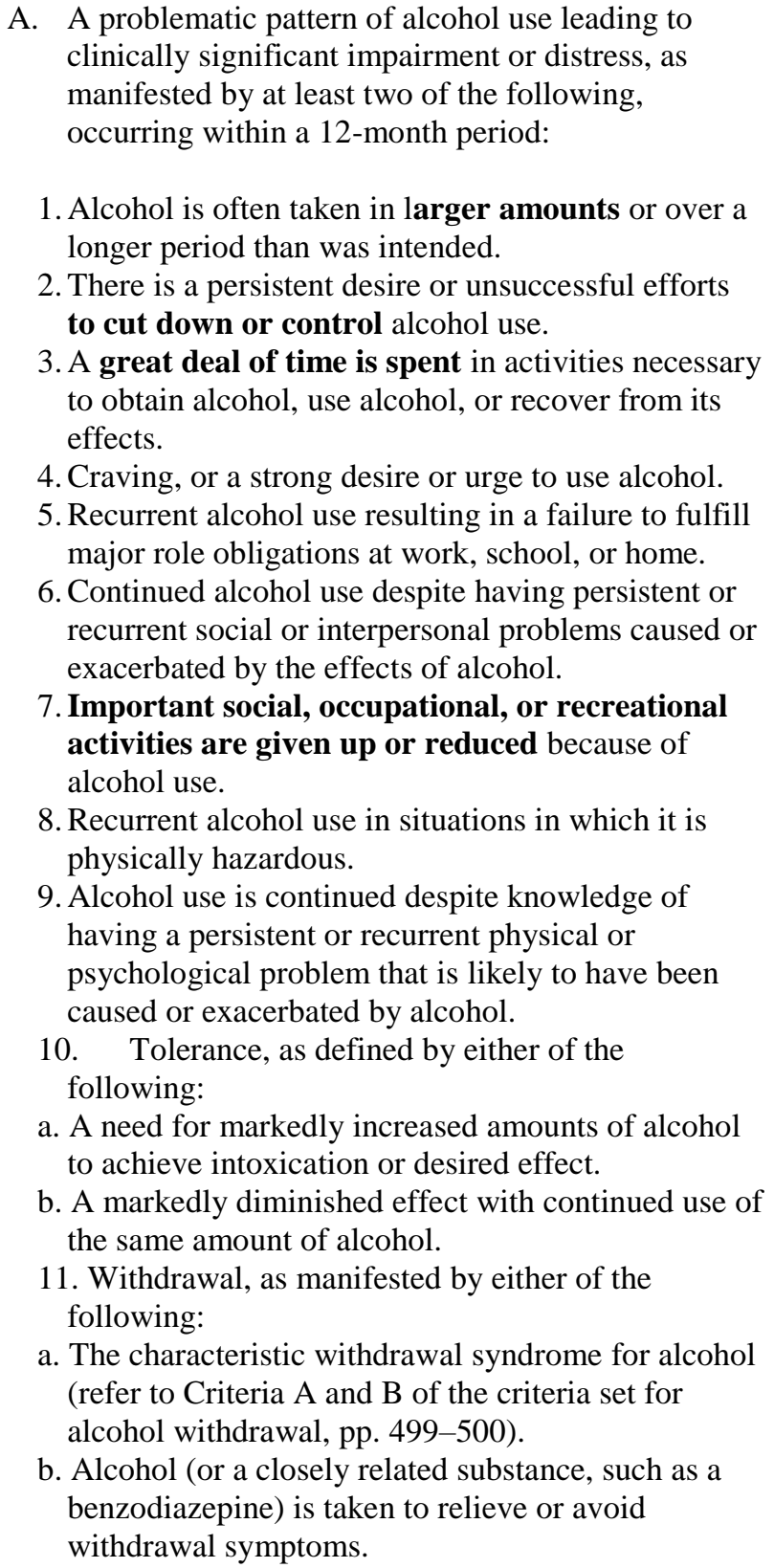 \\
\hline
\end{tabular}




\begin{tabular}{|c|c|}
\hline Gambling Disorder Criteria & Alcohol Use Disorder \\
\hline \multicolumn{2}{|l|}{$\begin{array}{l}\mathrm{B}, \text { The gambling behavior is not better explained by } \\
\text { a manic episode. }\end{array}$} \\
\hline \multirow[t]{2}{*}{$\begin{array}{l}\text { Specify if: } \\
\text { - Episodic: Meeting diagnostic criteria at more than } \\
\text { one-time point, with symptoms subsiding } \\
\text { between periods of gambling disorder for at least } \\
\text { several months. } \\
\text { - Persistent: Experiencing continuous symptoms, to } \\
\text { meet diagnostic criteria for multiple years. }\end{array}$} & $\begin{array}{l}\text { Specify if: } \\
\text { In a controlled environment: This additional specifier is } \\
\text { used if the individual is in an environment where access } \\
\text { to alcohol is restricted. }\end{array}$ \\
\hline & $\begin{array}{l}\text { Specify if: } \\
\text { - In early remission: After full criteria for alcohol use } \\
\text { disorder were previously met, none of the criteria for } \\
\text { alcohol use disorder have been met for at least } 3 \\
\text { months but for less than } 12 \text { months (with the } \\
\text { exception that Criterion A4, "Craving, or a strong } \\
\text { desire or urge to use alcohol," may be met). } \\
\text { - In sustained remission: After full criteria for alcohol } \\
\text { use disorder were previously met, none of the criteria } \\
\text { for alcohol use disorder have been met at any time } \\
\text { during a period of } 12 \text { months or longer (with the } \\
\text { exception that Criterion A4, "Craving, or a strong } \\
\text { desire or urge to use alcohol," may be met). }\end{array}$ \\
\hline $\begin{array}{l}\text { Specify current severity: } \\
\text { - Mild: } 4-5 \text { criteria met. } \\
\text { - Moderate: } 6-7 \text { criteria met. } \\
\text { - Severe: } 8-9 \text { criteria met. }\end{array}$ & $\begin{array}{l}\text { Specify current severity: } \\
\text { - } 305.00 \text { (F10.10) Mild: Presence of } 2-3 \text { symptoms. } \\
\text { - } 303.90 \text { (F10.20) Moderate: Presence of } 4-5 \\
\text { symptoms. } \\
\text { - } 303.90 \text { (F10.20) Severe: Presence of } 6 \text { or more } \\
\text { symptoms. }\end{array}$ \\
\hline \multicolumn{2}{|c|}{$\begin{array}{l}\text { Note. DSM-V Criteria summarized from the American Psychiatric Association (APA). (2015). Diagnostic and statistical } \\
\text { manual of mental disorders (6th ed.). Washington, DC: Author. Bold indicates similarity of criteria. }\end{array}$} \\
\hline
\end{tabular}

\section{Screening Brief Intervention and Referral to Treatment}

As noted earlier, there are five phases to interdisciplinary treatment from a clinical social work and clinical behavioral analysis perspective (Wilson \& Matthieu, 2015). The focus here is now on the first two phases of that model, the identification of clinically relevant target behaviors and the use of reliable and valid measurements. As one example of an evidence based process, the Substance Abuse and Mental Health Services Administration (2016) describes Screening, Brief Intervention, and Referral to Treatment (SBIRT) as a sequential assessment and referral approach to the delivery of early intervention and treatment to individuals with substance use disorders and those at risk of developing these disorders. Screening quickly assesses the severity of substance use using a standardized assessment instrument and identifies the appropriate level of treatment. Brief intervention focuses on increasing insight and awareness regarding substance use and eliciting motivation toward behavioral change. Referral to treatment provides those identified as needing more extensive treatment with access to specialty care. Gambling as 
an additional substance use disorder can be easily integrated into this protocol, with the addition of appropriate gambling screening measures (which will be discussed later) and community referrals located for gambling treatment.

\section{Protocols for Assessing PTSD and Substance Use Disorders in Veterans}

Clinical practice guidelines (CPG) are guiding principles that include evidence based treatment recommendations intended to enhance the quality of care that individuals receive. The guidelines are built upon the knowledge gained through systematic reviews and meta-analysis of evidence, and assessments regarding potential benefits and risks of alternative interventions. Clinicians utilize CPG's in order to apply the best available evidence supported interventions and to make informed clinical decisions (DVA/DoD, 2015; DVA/DoD, 2017). Additionally, CPG's often include decision aids or clinical tools that provide a flow chart, or an algorithm, aimed at selecting the most appropriate clinical intervention for a situation. As defined, a clinical algorithm is a diagram that utilizes a stepby-step decision tree approach in order to assist a clinician in the decision making process (DVA/DoD, 2015; DVA/DoD, 2017. Symbols are used to display each step in the process, and arrows connect numbered decision points that indicate the order and direction the steps in the decision process should be made. For example, "if symptoms $\mathrm{x}$ and y are present, then use intervention z". What follows are summaries of the CPG's utilized by the DVA/DoD for the management of PTSD and SUD.

The DVA/DoD Clinical Practice Guideline for Management of Substance Use Disorder (December, 2015) is designed to assist providers in managing or co-managing patients with SUD. The patient population of interest for this CPG is adults who are eligible for care in the VA and DoD healthcare delivery systems that includes veterans, as well as, deployed and non-deployed military service members. As such, it does not provide recommendations for the management of SUD in children or adolescents. This CPG includes an algorithm, a step-by-step decision tree, which is designed to facilitate understanding of the clinical pathway and decision making process used in management of SUD. Standardized symbols are used to display each step in the algorithm, and arrows connect the numbered boxes indicating the order in which the steps should be followed. The CPG includes Algorithm A: Screening and Treatment, and Algorithm B: Stabilization. Although GD is included in the SUD section of DSM V, GD is not included in this CPG. With regard to other co-occurring conditions, this CPG notes that "of those with an SUD diagnosis, 55-75\% also received diagnoses for PTSD or depression" (p. 9).

The DVA/DoD Clinical Practice Guideline for Management of PTSD (ver 2.0, 2010) is relevant to all healthcare professionals who are providing or directing treatment services to patients with a history of trauma exposure. Post-traumatic stress is one disorder along a spectrum of anxiety disorders that focus on the individual's stress reaction to the trauma. These disorders are arranged along a temporal axis, from Acute Stress Reaction, to Acute Stress Disorder, Acute PTSD, and Chronic PTSD. Each of these may be associated with serious mental and physical co-occurring conditions. Some trauma survivors may experience only a part of this spectrum, while others may progress through the entire range, requiring different types of treatment along this temporal sequence. Algorithms included in this CPG are Core Algorithm: Initial Evaluation and Triage, Algorithm A: Acute Stress 
Reaction/Disorder Prevention of PTSD, Algorithm B: Assessment and Diagnosis of PTSD. This CPG (p. 90) identifies a documented and strong relationship between co-occurring PTSD and SUD, and recommends that all individuals with a diagnosis of PTSD should receive comprehensive assessment for SUD. However, in this CPG as the one previous, gambling is not included.

\section{Screeners}

The complexity of symptoms associated with co-occurring disorders often make the task of diagnosis difficult resulting in people receiving treatment for one disorder and not another. However, if a problem is not identified, one can expect that it will not receive the appropriate clinical attention. The importance of screening for co-occurring disorders is difficult to overstate. The following section identifies assessment measures that are utilized in the domains of PTSD, hazardous drinking or active alcohol use disorders, and severity of gambling symptoms. The list in Table 2 is not exhaustive and yet includes some strong, well-established screening measures.

Table 2. Short Screening Instruments for PTSD, Alcohol, Drug, and Gambling Disorders

\begin{tabular}{|c|c|c|c|}
\hline Measure & $\#$ of items & Domain & Website \\
\hline $\begin{array}{l}\text { Primary Care PTSD } \\
\text { Screen (PC-PTSD- 5) }\end{array}$ & $\overline{5 \text {-item }}$ & PTSD & $\begin{array}{l}\text { Prins, A., Bovin, M.J., Smolenski, } \\
\text { D.J. et al., 2016; } \\
\text { http://www.ptsd.va.gov/professional/ } \\
\text { assessment/screens/pc-ptsd.asp }\end{array}$ \\
\hline AUDIT-C & 3-item & $\begin{array}{l}\text { Hazardous drinkers } \\
\text { or active alcohol } \\
\text { use disorders }\end{array}$ & $\begin{array}{l}\text { http://www.integration.samhsa.gov/i } \\
\text { mages/res/tool_auditc.pdf }\end{array}$ \\
\hline DAST-10 & 10-items & $\begin{array}{l}\text { Drug use (not } \\
\text { including alcohol) }\end{array}$ & $\underline{\text { http://www.integration.samhsa.gov/cl }}$ \\
\hline $\begin{array}{l}\text { Brief Biosocial } \\
\text { Gambling Screen } \\
\text { (BBGS) }\end{array}$ & 3-item & Gambling & Contact author. \\
\hline $\begin{array}{l}\text { South Oaks Gambling } \\
\text { Screen (SOGS) }\end{array}$ & 20-item & Gambling severity & $\begin{array}{l}\text { http://www.ncrg.org/sites/default/file } \\
\text { s/uploads/docs/monographs/sogs.pdf }\end{array}$ \\
\hline \multicolumn{4}{|c|}{$\begin{array}{l}\text { Note. Many assessment measures can be obtained at the National Center for PTSD by completing } \\
\text { the request form located at http://www.ptsd.va.gov/professional/assessment/ncptsd-instrument- } \\
\text { request-form.asp }\end{array}$} \\
\hline
\end{tabular}

The Primary Care PTSD Screen (PC-PTSD; Prins et al., 2003), is a 4-item screener that was designed for use in primary care and other medical settings, and is currently used to screen for PTSD in veterans using VA health care. The screen includes an introductory sentence to cue respondents to traumatic events and asks about four main clusters of symptoms. A DSM V revised version of the PC-PTSD has been developed with 5 items (Prins et al., 2016) and will be available from the NC-PTSD website once it is validated and ready for wide scale dissemination (NC-PTSD, 2016).

The AUDIT-C (Bush, Kivlahan, McDonell, et al., 1998) is a three item alcohol screener that can help identify persons who are hazardous drinkers or have active alcohol 
use disorders. The AUDIT-C is a modified version of the 10 question AUDIT instrument and is available for use in the public domain.

DAST-10 (Skinner, 1982) is a 10 item screener related to an individuals' possible involvement with drugs, not including alcoholic beverages, during the past 12 months which is adapted from the full 28 item DAST screen. "Drug abuse" refers to (1) the use of prescribed or over-the-counter drugs in excess of the directions, and (2) any nonmedical use of drugs. The various classes of drugs may include cannabis (marijuana, hashish), solvents (e.g., paint thinner), tranquilizers (e.g., Valium), barbiturates, cocaine, stimulants (e.g., speed), hallucinogens (e.g., LSD) or narcotics (e.g., heroin).

The Brief Biosocial Gambling Screen (BBGS; Gebauer, LaBrie, \& Shaffer, 2010) is a 15-item screening tool targeting past year experiences of withdrawal, deception, and bailout related to gambling behaviors. To date, no research has been conducted on the clinical validity or reliability (see also Volberg \& Williams, 2011). Gambling proclivity is assessed using the South Oaks Gambling Screen (SOGS; Lesieur \& Blume, 1987), where scores of 5 or higher indicate disordered or pathological gambling. Gambling severity is assessed using the Gambling Severity Assessment Scale (GSAS). Function of gambling play is also assessed using the Gambling Functional Assessment II (GFA-II; Dixon, Wilson, Belisle, \& Schrieber, under review).

Table 3. Evidence Based Treatments Recommended in Publically Available Clinical Practice Guidelines for Trauma, Substance Use and Gambling Disorders

\begin{tabular}{|c|c|c|}
\hline Domain & Treatment & Publically Available Clinical Practice Guidelines \\
\hline$\overline{\text { PTSD }}$ & $\begin{array}{l}\text { Prolonged Exposure } \\
\text { Cognitive Processing } \\
\text { Therapy } \\
\text { Eye Movement } \\
\text { Desensitization and } \\
\text { Reprocessing } \\
\text { Stress Inoculation Therapy }\end{array}$ & 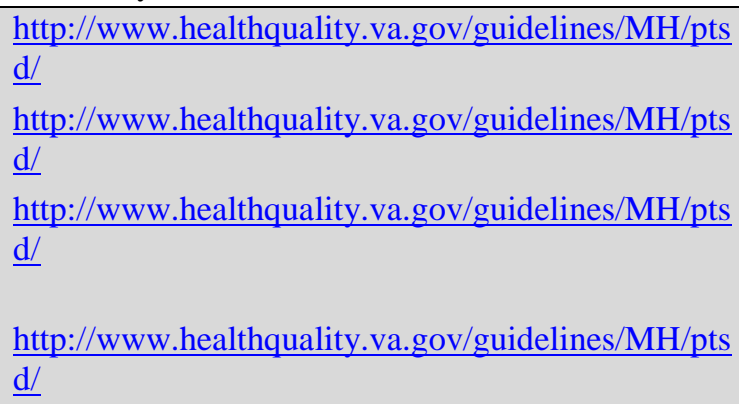 \\
\hline SUD & $\begin{array}{l}\text { Cognitive-Behavioral } \\
\text { Coping Skills Therapy } \\
\text { Community Reinforcement } \\
\text { Approach } \\
\text { Motivational Enhancement } \\
\text { Therapy }\end{array}$ & $\begin{array}{l}\text { http://www.healthquality.va.gov/guidelines/mh/sud/ } \\
\text { index.asp } \\
\text { http://www.healthquality.va.gov/guidelines/mh/sud/ } \\
\text { index.asp } \\
\text { http://www.healthquality.va.gov/guidelines/mh/sud/ } \\
\text { index.asp }\end{array}$ \\
\hline Gambling & $\begin{array}{l}\text { Cognitive Behavioral } \\
\text { Therapy } \\
\text { Acceptance and } \\
\text { Commitment Therapy }\end{array}$ & $\begin{array}{l}\text { https://store.samhsa.gov/shin/content/PGKIT- } \\
\text { 07/PGKIT-07-03.pdf } \\
\text { https://www.guideline.gov/summaries/summary/39 } \\
\underline{327}\end{array}$ \\
\hline
\end{tabular}

Note. A listing of all current and updated DVA/DoD Clinical Practice Guidelines can be found at http://www.healthquality.va.gov 


\section{Overview of Evidence Based Treatments for Veterans}

As noted in Table 3, there are a variety of evidence based treatments for PTSD, substance use, and gambling disorders, noted in the DVA/DoD Clinical Practice Guidelines for PTSD and substance use. Given that veterans have high rates of co-occurring PTSD and substance use disorders, the guidelines recommend treatments for each condition separately. However, in both of these guidelines, when conditions of PTSD and substance use co-occur, the recommendations include standardized assessments of both conditions and using a shared decision making process to prioritize care when recovery is emerging. Yet in both, gambling is not included as a behavioral addiction or as a SUD. The following section will review specific treatments that are evidence based for PTSD and substance use and recommended by the guidelines for veterans.

\section{PTSD Treatments}

Clinical practice guidelines for PTSD recommend four psychotherapies as having good evidence for effectiveness: Prolonged Exposure (PE), Cognitive Processing Therapy (CPT), Eye Movement Desensitization and Reprocessing (EMDR), and Stress Inoculation Training (SIT) (DVA/DoD, 2017). VHA policies and implementation efforts for PTSD have focused particularly on CPT and PE because they had the strongest evidence base at the time, including at least one study with veterans (Karlin et al., 2010). As a quick overview, both CPT and PE are manualized, trauma-focused cognitive-behavioral therapies that address and treat the cognitive and behavioral symptoms of PTSD, with improvements also seen in other outcomes such as depression and anxiety (Haagen et al., 2015). CPT typically includes 10-15 sessions lasting 60 minutes if delivered individually or 90 minutes if delivered in a group. It is broadly divided into three main parts: 1) PTSD symptom overview and psychoeducation, 2) the cognitive triad, and 3) examining and challenging beliefs related to the trauma and its impact. PE is only provided in 90-minute individual sessions and the number of sessions range from 8-15 sessions. The treatment consists of four main components: 1) treatment rationale and psychoeducation, 2) breathing retraining, 3) in vivo exposure, and 4) imaginal exposure.

\section{Substance Use Treatments}

Clinical practice guidelines recommend the following interventions for substance use as having good evidence for effectiveness: Cognitive-behavioral coping skills training, community reinforcement approach, and motivational enhancement therapy. Cognitivebehavioral coping skills therapy consists of "related treatment approaches for substance use that focus on teaching patients to modify both thinking and behavior related not only to substance use, but to other areas of life functionally related to substance use" (DVA/DoD, 2015, p. 92). Individuals learn to identify thoughts and actions that have behavioral consequences that relate to and reinforce substance use. As a way to interrupt a problematic relationship between their thoughts and behaviors, individuals acquire skills aimed at "changing thinking and behaviors that contribute to substance use, and to strengthen coping skills, improve mood, interpersonal functioning and enhance social support" (VA/DOD, December, 2015, p. 92) 
Environmental factors can impact and influence an individual's behavior. Community reinforcement approach (CRA) "is a comprehensive cognitive-behavioral intervention for the treatment of substance use by focusing on environmental contingencies that impact and influence the patient's behavior" (VA/DOD, December, 2015, p. 92). Based upon the assertion that the environmental factors play a key role in additive behavior, CRA utilizes changes in natural environmental supports, such as family, social, and occupational, to enhance the opportunity to change drinking/using behaviors.

An individual's motivation to change is an important indicator of success with regard to behavior change. Motivational enhancement therapy (MET) is based on "principles of motivational interviewing (MI) including an empathic, client-centered, but directive, approach intended to heighten awareness of ambivalence about change, promote commitment to change, and enhance self-efficacy." (VA/DOD, December, 2015, p. 92). Effectively enhancing an individual's willingness to effect change in their behavior can greatly increase the effectiveness of and commitment to an individualized recovery plan. Overall, these SUD treatments draw heavily from behavior theories and utilize strategies for specifically targeted behavioral change.

\section{Gambling Treatments}

Selecting an evidence-based intervention includes the integration of best available research with clinical expertise in the context of client characteristics, culture, and preferences (APA, 2015). Meta-analyses of gambling treatment have found the effects of psychotherapeutic interventions to have positive outcomes (Pallesen et al., 2005; Cowlishaw et al., 2012). For example, CBT (Gooding \& Tarrier, 2009) has proven effective in reducing problem gambling with significant effect sizes across $3,6,12$, and 24 month follow up. Overall success rates for gambling treatments are estimated at $70 \%$ for six months follow up and 50\% for one year follow up (Lopez-Viets, \& Miller, 1997). Utilizing CBT as a therapeutic intervention, and internet based CBT (Casey et al., 2017), to treat gambling disorder typically involves three targets that include 1) identifying and changing cognitive distortions about gambling, 2) reinforcing non-gambling behaviors, 3) and recognizing positive and negative consequences (Petry, 2009). Additionally, emerging evidence suggests mindfulness-based interventions, such as acceptance and commitment therapy, for disordered gambling demonstrate positive and significant effects on gambling behavior and symptoms, urges, and financial outcomes (Maynard, Wilson, Labuzienski, \& Whiting, 2015).

The goal of ACT is to enhance an individual's psychological flexibility. ACT targets psychological flexibility through the use of six interconnected processes that include acceptance, present moment focus, diffusion, self as context, committed action, and values (Dixon, Wilson, \& Habib, 2016). The empirical evidence in support of ACT as an effective therapeutic technique continues to expand (Maynard et al., 2015; Dixon et al., 2016), and has been evidence of an effective treatment option for substance use disorders (Dixon et al., 2016). Both, CBT and ACT, are effective interventions with regard to treating the behavioral symptoms presented in GD. 
GD is very likely under-idenified by mental health clinicians, as well as those in the community, bearing in mind the high rates of co-occurring disorders associated with GD. Edens and Rosenheck (2012) concluded that in a sample of Veterans Affairs (VA) specialty mental health services users $(\mathrm{N}=1,102,846)$ was indicative of GD under-diagnosis with a rate of six times below national estimates. Given serious associated co-occurring conditions, increased awareness of this condition among mental health clinicians could facilitate referral and utilization of effective services and timely access to treatment (Edens \& Rosenheck, 2012).

Although a significant association between GD and other disorders have been identified, the GD literature has largely overlooked the importance of trauma and posttraumatic stress disorder (PTSD) as a prevalent co-occurring condition (Ledgerwood \& Milosevic, 2015). In fact, those with lifetime PTSD also were more likely to use gambling as a way to cope with negative emotions and experienced greater negative emotionality (Ledgerwood \& Milosevic, 2015). PTSD is a prevalent condition in many subpopulations such as, women who have experienced sexual assault, and veterans, and is associated with greater psychiatric co-morbidity. Considering that PTSD is not an uncommon diagnosis in the veteran community, effective assessment and treatment of concurrent GD and PTSD is crucial to providing appropriate health care services to veteran population.

Given a similarity in functional response, research has identified a compelling association between posttraumatic stress disorder (PTSD) and Gambling Disorder (GD). The National Comorbidity Study Replication identified that $14.8 \%$ of those with lifetime GD met criteria for lifetime PTSD (Kessler et al., 2008). Overall, co-occurring disorders often have a larger impact on individuals when compared to those with only one of the problem areas. As examples, individuals who experience increased depressive symptoms (Taber, McCormick, \& Ramirez, 1987), engage in gambling at a younger age, experience more severe gambling problems (Petry \& Steinberg, 2005), and experience greater psychiatric symptom severity, impulsivity, and dissociation (Ledgerwood \& Petry, 2006). Amid this strong and growing evidence base on GD, to our knowledge, there is a compelling lack of guidance, inclusion, or discussion of GD in clinical practice guidelines nor co-occurring treatments for the veteran population. While glaring, this omission is an opportunity to reinforce the interdisciplinary treatment model suggested by clinical social work and behavioral analysis.

\section{Interdisciplinary Treatment Model}

As noted previously, there are five areas of interdisciplinary social work and behavior analytic practice (Wilson \& Matthieu, 2015) to assist clinicians with case conceptualization: 1) identification of clinically relevant target behaviors; 2) use of reliable and valid measurements; 3) relationship between target behaviors, measures, and treatment; 4) data-based treatment decisions; and 5) social validity of treatment process and outcomes. In the following case example, we highlight the use of this process model using the first two steps. 


\section{Case Example}

Cindy was a 42-year-old African American female with chronic pain, rheumatoid arthritis, and a neck injury from her service in the Army. She was a wife and mother to her only son, who was 21-years-old. She worked in an administrative position for the Department of Defense, and took pride in her job still being connected to serving her country. She reported recent mood swings, nightmares, and intrusive thoughts related to a sexual assault that occurred during her military service. Currently, she reported that her and her husband spent more and more time at the local casino, as a way to spend time with each other and to get out of the house. Eventually, she was trading in her car title for quick cash, and went through the family savings within 6 months. When Cindy self-referred for gambling services, her family did not know of her condition, and she had gambled away two cars, countless household items, and was unable to pay her mortgage. A retrospective baseline of her gambling the 60 days prior to her intake, identified Cindy had gambled for 35 days in the casino (slot machines), over 6 hours per visit, and had wagered over $\$ 10,000$.

During screening, she was identified as having severe gambling disorder (South Oaks Gambling Screen (SOGS; Lesieur \& Blume, 1987) score of 18), and gambled to access escape and tangible items according to the Gambling Functional Assessment (GFA; Dixon \& Johnson, 2007). During treatment, she was concerned with her gambling behaviors. According to the PC-PTSD screener (Prins et al., 2003), Cindy scored a 4 (yes to all 4 items) and then completed the self-report Post Traumatic Stress Disorder (PTSD) Checklist -Military Version (PCL-M; Blanchard, Jones-Alexander, Buckley, \& Forneris, 1996). Cindy met criteria for PTSD and scored in the clinical range on the PCL with a score of 45 , and endorsed a number of PTSD symptoms in the extreme range in the month prior (e.g., daily and nightly repeated, disturbing memories, thoughts, or images of a stressful experience from the military; avoiding thinking, talking, or similar activities or situations that remind her of her military experience; and feeling jumpy or easily startled, or being "super alert" or watchful and on guard). Clinical interviews were then completed to identify functional relations between Cindy's co-occurring symptoms and environmental events. SBIRT assessment using the AUDIT-C revealed that Cindy was in the risky range of substance use with a score of 10 and was proved patient education materials and referred to treatment. Therefore, Cindy's target behaviors included gambling, trauma related symptoms and alcohol use. Trauma treatment, specifically PE was initiated to address the trauma symptoms while initiating treatment for gambling disorders to include Acceptance and Commitment Therapy. Referrals to self-help programs for substance use were also provided.

\section{Resources for Practice}

There are a host of available materials related to the training of interdisciplinary treatment teams on trauma and addictions. However, more limited are publically available resources and those specifically on behavioral additions such gambling and targeted to veterans. Therefore, here we highlight a number of these resources and offer additional resources to support interdisciplinary clinical practice in Table 2 and Table 3. 
The Substance Abuse and Mental Health Administration (SAMHSA) has invested heavily in disseminating the evidence based treatment protocol entitled Screening, Brief Intervention and Referral to Treatment. At present, there is an SBIRT core curriculum that is publically available by request for the CD-ROM from SAMHSA. There are also a number of free publically available online SBIRT training courses listed on the SAMHSAHRSA Center for Integrated Health Solutions. Some additional particularly easy to navigate and free online SBIRT courses are also available online (See Table 4).

Table 4. Publically Available Resources to Support Clinical Practice.

\begin{tabular}{|c|c|c|}
\hline Topic & Description & Publically Available Resource \\
\hline \multirow[t]{3}{*}{ SBIRT } & CD-ROM & http://www.samhsa.gov/sbirt \\
\hline & Online Training Course & $\begin{array}{l}\text { http://www.integration.samhsa.gov/clinical- } \\
\text { practice/sbirt/training-other-resources }\end{array}$ \\
\hline & Online Courses & $\begin{array}{l}\text { http://healtheknowledge.org/course/index.php?categ } \\
\underline{\text { oryid=50. }}\end{array}$ \\
\hline \multirow[t]{3}{*}{ PTSD } & $\begin{array}{l}\text { Assessment Measures } \\
\text { Online Request Form }\end{array}$ & $\begin{array}{l}\text { http://www.ptsd.va.gov/professional/assessment/nc } \\
\text { ptsd-instrument-request-form.asp. }\end{array}$ \\
\hline & $\begin{array}{l}\text { DVA/DoD Clinical } \\
\text { Practice Guidelines }\end{array}$ & http://www.healthquality.va.gov \\
\hline & $\begin{array}{l}\text { Community Provider } \\
\text { Toolkit }\end{array}$ & $\begin{array}{l}\text { http://www.mentalhealth.va.gov/communityprovide } \\
\underline{\text { rs/clinic sud.asp. }}\end{array}$ \\
\hline \multirow[t]{2}{*}{ SUD } & $\begin{array}{l}\text { DVA/DoD Clinical } \\
\text { Practice Provider, Family } \\
\text { and Patient Resources }\end{array}$ & $\underline{\text { http://www.healthquality.va.gov/guidelines/MH/sud }}$ \\
\hline & $\begin{array}{l}\text { Self-Help Toolkit: The 3- } \\
\text { step Referral Method }\end{array}$ & $\begin{array}{l}\text { http://www.mentalhealth.va.gov/providers/sud/selfh } \\
\text { elp/ }\end{array}$ \\
\hline
\end{tabular}

With regard to assessing and treating PTSD, the VA's National Center for PTSD hosts a list of assessment measures that can be obtained by completing the online request form. For practice guidelines, there are a variety of DVA/DoD Clinical Practice Guideless that are publically available for download, and can be found on the VA Health Quality website. For each guideline, there are free downloadable additional tools for the provider, family, and patient resources, such as the one for substance use noted in Table 4. One substance use related link in the online resources is to a particularly good Self-Help Toolkit: The 3step Referral Method. Finally, the VA disseminates many of the mental health products, trainings and resources available on a community provider's website, called the Community Provider Toolkit. This website offers a military culture training, mini clinic topics on couples and family, disability benefits, posttraumatic stress disorder, serious mental illness, smoking and tobacco use, substance use, suicide prevention, technology in care, and women veterans, and other clinician friendly tools and handouts.

\section{Discussion}

The use of an interdisciplinary model can assist clinicians in the identification of appropriate evidence-based assessments and treatments for co-occurring PTSD and 
substance use disorders. A case example focused on the use of specific assessment tools and treatment approaches drawn from DVA/DoD clinical practice guidelines demonstrate how clinicians can use the model in practice, to identify target behaviors, valid screening measures, and effective behavior-based treatments. Finally, resources related to the training of interdisciplinary treatment teams on trauma and addictions, particularly for behavioral additions such gambling, was presented. By noting the exclusion of GD, we strongly hope that the future will bring an integration of gambling as a behavioral addiction into the evidence-based practices of clinicians who assess and treat co-occurring conditions.

\section{Implications for Practice with Veterans and VA}

Recent changes to the DSM has re-categorized gambling as a substance use disorder. For the VA, this change may instigate major national service delivery changes with substance use disorder treatment for veterans to now include gambling treatment and services within the existing "minimum requirements" services mandate (DVA, 2008). As outlined in VHA Handbook 1160.01, Uniform Mental Health Services in VA Medical Centers and Clinics, this document requires VA facilities to offer or to contract out to the community substance use treatment for all Veterans with a clinical need. At present, to our knowledge, gambling addiction is not addressed in any of the VA's mandatory national screening and treatment requirements. To determine if pathological gambling is currently an unmet need in the VA, the National Council on Problem Gambling recently recommended a two-part study of all VA patients to determine the severity of gambling problems and to determine if the VA health care system has the capacity to serve this population (Newhouse, 2013). Until this national study is completed, it is critical to assess local services for the capacity for referrals and the barriers and facilitators to veterans receiving evidence based assessments and treatments for gambling.

With the advent of new changes to the DSM V and the requirements for SUD treatments provided by the VA health care system, there are various individual, provider and system level factors that may limit the extent to which gambling treatment options are offered. First, military service members may face disciplinary action (e.g., demotion) for seeking help while still serving in the armed forces (Emshoff, Gilmore, \& Zorland, 2010). Secondly, the extent to which VA and military providers are systematically identifying atrisk or disordered gamblers is unknown (Whiting et al., 2016).

Lastly, system level accessibility issues are also unknown. These issues include the frequency of gambling treatment referrals from national gambling helplines (1-800-5224700) or local crisis lines for veterans (1-800-273-8255 and Press 1 for military or veteran) to the few existing out of state VA facilities with gambling treatment services (e.g., VA New England Health Care System, Louis Stokes Cleveland Health Care System), to local gambling treatment programs or specialty behavioral health providers such as social workers and applied behavior analysts in the community, or to self-help groups (e.g. Gamblers Anonymous). Given that co-occurring addictions treatment for veterans may occur in the VA for PTSD and SUD, and more commonly in the community with specialized gambling treatment programs or private practitioners, this fragmented system of care will require an interdisciplinary, collaborative, case management approach. Collecting information within local communities and in the VA to assess the availability 
of gambling treatment services is the first step in determining the local accessibility and then the quality of services for military and Veterans. While there is an emerging picture of the etiology and prevalence of gambling in general (Petry et al., 2005) and among Veterans and military personnel specifically (Newhouse, 2013) it is still unclear the extent to which clinicians in the community or VA are identifying and treating disordered or atrisk gamblers.

\section{Conclusion}

Co-occurring mental health and substance use issues can present challenges to clinicians in recognizing both disorders and obtaining utilizing the best intervention for individuals. Limited evidence exists to guide interdisciplinary treatment teams in effective behavioral treatment of co-occurring PTSD and GD. The aim of the article was to begin to address that gap in literature. We provided a model for interdisciplinary practice to assist social work, behavioral analysis, and other clinicians working with addictions and traumarelated symptoms in selecting appropriate evidence based assessments and treatments. As respective professions continuously move to enhance client outcomes in behavioral health, the importance of interdisciplinary treatment becomes increasingly vital.

\section{References}

American Psychiatric Association [APA]. (2015). Diagnostic and statistical manual of mental disorders (6th ed.). Washington, DC: Author.

Association for Behavior Analysis International. (2017). What is Behavioral Analysis? Retrieved from: https://www.abainternational.org/about-us/behavior-analysis.aspx

Blanchard, E. B., Jones-Alexander, J., Buckley, T. C., \& Forneris, C.A. (1996). Psychometric properties of the PTSD Checklist (PCL). Behavior Research and Therapy, 34, 669-673

Bolen, D. W, \& Boyd, W. H. (1968). Gambling and the gambler: A review and preliminary findings. Archives of General Psychiatry, 18, 617-630. doi: https://doi.org/10.1001/archpsyc.1968.01740050105018

Bush K., Kivlahan, D. R., McDonell M. B., Fihn, S. D., \& Bradley, K. A. (1998). The AUDIT alcohol consumption questions (AUDIT-C): An effective brief screening test for problem drinking. Ambulatory Care Quality Improvement Project (ACQUIP). Archives of Internal Medicine, 158, 1789-1795. doi: https://doi.org/10.1001/archinte.158.16.1789

Casey, L. M., Oei, T. P., Raylu, N., Horrigan, K., Day, J., Ireland, M., \& Clough, B. A. (2017). Internet-based delivery of cognitive behaviour therapy compared to monitoring, feedback and support for problem gambling: A randomised controlled trial. Journal of Gambling Studies [online], 1-18. doi: https://doi.org/10.1007/s10899-016-9666-y

Cowlishaw, S., Merkouris, S., Dowling, N., Anderson, C., Jackson, A., \& Thomas, S. (2012). Psychological therapies for pathological and problem gambling. The Cochrane Database of Systematic Reviews, 11CD008937. doi: https://doi.org/10.1002/14651858.CD008937.pub2 
Department of Veterans Affairs, Department of Defense [DVA/DoD]. (2015). DVA/DoD Clinical Practice Guideline for Management of Substance Use Disorder: Guideline Summary (ver.3.0). Office of Quality and Performance. Washington DC: Author.

Department of Veterans Affairs, Department of Defense [DVA/DoD]. (2017). DVA/DoD Clinical Practice Guideline for Post Traumatic Stress Disorder and Acute Stress Reaction. (ver. 3.0). Office of Quality and Performance. Washington DC: Author.

Dixon, M. R., \& Johnson, T. E. (2007). The gambling functional assessment (GFA): An assessment device for identification of the maintaining variables of pathological gambling. Analysis of Gambling Behavior, 1(1), 44-49.

Dixon, M. R., Wilson, A. N., \& Habib, R. (2016). Empirical research: Neurological evidence of acceptance and commitment therapy effectiveness in college-age gamblers. Journal of Contextual Behavioral Science, 5(2), 580-588. doi: https://doi.org/10.1016/j.jcbs.2016.04.004

Dixon, M. R., Wilson, A. N., Belisle, J., \& Schreiber, J. (under review). Assessing the construct validity of the gambling functional assessment. The Psychological Record.

Edens, E., \& Rosenheck, R. (2012). Rates and correlates of pathological gambling among VA mental health service users. Journal of Gambling Studies, 28(1), 1-11. doi: https://doi.org/10.1007/s10899-011-9239-Z

Emshoff, J., Gilmore, D., \& Zorland, J. (2010). Veterans and problem gambling: A review of the literature. Georgia State University. Retrieved from http://www2.gsu.edu/ psyige/Rsrc/PG_IPV_Veterans.pdf

Gebauer, L., LaBrie, R. A., \& Shaffer, H. J. (2010). Optimizing DSM-IV-TR classification accuracy: A brief bio-social screen for detecting current gambling disorders among gamblers in the general household population. Canadian Journal of Psychiatry, 55(2), 82-90. doi: https://doi.org/10.1177/070674371005500204

Gooding P.A. \& Terrier. N., (2009). A systematic review and meta-analysis of cognitivebehavioural interventions to reduce problem gambling: Hedging our bets? Behaviour Research and Therapy, 47, 592-607 doi: https://doi.org/10.1016/j.brat.2009.04.002

Karlin, B. E., Ruzek., J. I., Chard, K. M., Eftekhari, A., Monson, C. M., Hembree, E. A., . . . Foa, E. B. (2010). Dissemination of evidence-based psychological treatments for posttraumatic stress disorder in the Veterans Health Administration. Journal of Traumatic Stress, 23, 663-673. doi: https://doi.org/10.1002/jts.20588

Kessler, R. C., Hwang, I., Labrie, R., Petukhova, M., Sampson, N. A., Winters, K. C., . . (2008). DSM-IV pathological gambling in the National Comorbidity Survey Replication. Psychological Medicine, 38, 1-10. doi: https://doi.org/10.1017/S0033291708002900

Ledgerwood, D., \& Milosevic, A. (2015). Clinical and personality characteristics associated with post-traumatic stress disorder in problem and pathological gamblers recruited from the community. Journal of Gambling Studies, 31(2), 501-512. doi: https://doi.org/10.1007/s10899-013-9426-1 
Ledgerwood, D. M., \& Petry, N. M. (2006). Posttraumatic stress disorder symptoms in treatment-seeking pathological gamblers. Journal of Traumatic Stress, 19, 411-416. doi:https://doi.org/10.1002/jts.20123

Lesieur, R., \& Blume, S. (1987). The South Oaks Gambling Screen (SOGS): A new instrument for the identification of pathological gamblers. American Journal of Psychiatry, 144, 1184-1188. doi: https://doi.org/10.1176/ajp.144.9.1184

Lopez-Viets, V. C., \& Miller, W. R. (1997). Treatment approaches for pathological gamblers. Clinical Psychology Review, 17, 689-702

Maynard, B. R., Wilson, A. N., Labuzienski, E., \& Whiting, S. W. (2015). Mindfulness-based approaches in the treatment of disordered gambling: A systematic review and metaanalysis. Research on Social Work Practice [online]. doi:

https://doi.org/10.1177/1049731515606977

National Association of Social Workers [NASW]. (1989). NASW Standards for the Practice of Clinical Social Work. Washington, DC: NASW Press.

Newhouse, J. (2013). Vets vulnerable to gambling addictions. Psychology Today. Retrieved from: ww.psychologytoday.com/blog/invisible-wounds/201305/vets-vulnerablegambling-addictions.

Pallesen, S., Mitsem, M., Kvale, G., Johnsen, B., \& Molde, H. (2005). Outcome of psychological treatments of pathological gambling: a review and metaanalysis. Addiction, 100, 1412-1422. doi: https://doi.org/10.1111/j.13600443.2005.01204.X

Petry, N. M. (2009). Disordered gambling and its treatment. Cognitive and Behavioral Practice, 16(4), 457-446. doi: https://doi.org/10.1016/j.cbpra.2009.02.005

Petry, N. M., Stinson, F. S., \& Grant, B. F. (2005). Comorbidity of DSM-IV pathological gambling and other psychiatric disorders: Results from the national epidemiologic survey on alcohol and related conditions. Journal of Clinical Psychiatry, 66, 564-574. doi: https://doi.org/10.4088/JCP.v66n0504

Petry, N. M., \& Steinberg, K. L. (2005). Childhood maltreatment in male and female treatment-seeking pathological gamblers. Psychology of Addictive Behaviors, 19, 226229. doi: https://doi.org/10.1037/0893-164X.19.2.226

Prins, A., Ouimette, P., Kimerling, R., Cameron, R. P., Hugelshofer, D. S., Shaw-Hegwer, J...Sheikh, J. I. (2003). The Primary Care PTSD Screen (PC-PTSD): Development and operating characteristics. Primary Care Psychiatry, 9, 9-14. doi:

https://doi.org/10.1185/135525703125002360

Prins, A., Bovin, M.J., Smolenski, D.J., Marx, B. P., Kimerling, R., Jenkins-Guarnieri, M. A. ... Tiet, Q. Q. (2016). The primary care PTSD screen for DSM-5 (PC-PTSD-5): Development and evaluation within a veteran primary care sample. Journal of General Internal Medicine, 31(10), 1206-1211. doi: https://doi.org/10.1007/s11606-016-3703-5

Raylu, N., \& Oei, T. P. (2002). Pathological gambling. A comprehensive review. Clinical Psychology Review, 22, 1009-1061. doi: https://doi.org/10.1016/S0272-7358(02)00101-0 
Substance Abuse and Mental Health Services Administration [SAMHSA]. (2016). SBIRT: Screening, brief intervention, and referral to treatment. Retrieved from http://www.samhsa.gov/sbirt

SAMHSA-HRSA Center for Integrated Health Solutions. (n.d.). AUDIT-C - Overview. Retrieved from: http://www.integration.samhsa.gov/images/res/tool_auditc.pdf

SAMHSA-HRSA Center for Integrated Health Solutions. (n.d.). Screening tools: DAST-10. Retrieved from: http://www.integration.samhsa.gov/clinical-practice/screening-tools

Skinner H. (1982). The Drug Abuse Screening Test. Addictive Behaviors, 7, 363-371. doi: https://doi.org/10.1016/0306-4603(82)90005-3

Taber, J. I., McCormick, R. A., \& Ramirez, L. F. (1987). The prevalence and impact of major life stressors among pathological gamblers. International Journal of the Addictions, 22, 71-79. doi: https://doi.org/10.3109/10826088709027414

U.S. Department of Veterans Affairs [DVA]. (2008). VHA Handbook 1160.01 Uniform Mental Health Services in VA Medical Centers and Clinics. Washington, D.C.: Author.

U.S. Department of Veteran Affairs: National Center for PTSD [NC-PTSD]. (2016). Primary care PTSD screen (PC-PTSD). Retrieved from http://www.ptsd.va.gov/professional/assessment/screens/pc-ptsd.asp

Volberg, R. A., \& Williams, R. J. (2011). Developing a brief problem gambling screen using clinically validated samples of at-risk, problem and pathological gamblers. Report to the Alberta Gaming Research Institute. Retrieved from: https://www.uleth.ca/dspace/bitstream/handle/10133/2561/2011-Brief\%20ScreenAGRI.pdf?sequence $=1$ \&isAllowed $=y$

Whiting, S. W., Potenza, M. N., Park, C. L., McKee, S. A., Mazure, C. M., \& Hoff, R. A. (2016). Investigating veterans' pre-, peri-, and post-deployment experiences as potential risk factors for problem gambling. Journal of Behavioral Addictions 5(2), 213-220. doi: https://doi.org/10.1556/2006.5.2016.027

Wilson, A. N., \& Matthieu, M. M. (2015). Clinical application of behavior analytic social work practice. In H. S. Roane, J. L. Ringdahl, \& T. S. Falcomata (Eds.), Clinical and organizational applications for applied behavior analysis (pp. 501-522). London: Elsevier.

Author note: Address correspondence to: Monica M. Matthieu, PhD, LCSW, School of Social Work, College for Public Health and Social Justice, 3550 Lindell Blvd., Saint Louis University, Saint Louis. MO 63103. 